\title{
When bugs and drugs conspire: driving acneiform skin toxicity
}

\author{
Allison C. Billi, Mrinal K. Sarkar, and Johann E. Gudjonsson \\ Department of Dermatology, Michigan Medicine, University of Michigan, Ann Arbor, Michigan, USA.
}

\begin{abstract}
Therapy with antineoplastic agents that inhibit EGFR and MEK is frequently limited by cutaneous adverse reactions, most commonly acne-like eruptions. In this issue of the $J C l$, Satoh et al. define a mechanism for acneiform skin toxicity wherein EGFR/MEK inhibitors cooperate with the skin commensal Cutibacterium acnes to induce IL-36 $\gamma$ in keratinocytes via the combined actions of Krüppel-like factor 4 and NF-KB transcription factors at the IL-36 $\gamma$ promoter, resulting in neutrophil recruitment. In addition to elucidating why EGFR/MEK inhibitor-induced rashes are often pustular and folliculocentric, this mechanism provides justification for the long-standing practice of management with antibiotic therapy.
\end{abstract}

by pilosebaceous units, the authors speculated that EGFRis may work cooperatively with $C$. acnes, which abundantly colonizes sebum-rich skin and plays a pathogenic role in acne via TLR2 signaling, which in turn activates NF- $\mathrm{B}(5)$. Exposing primary human keratinocytes to $C$. acnes and EGFR inhibition via drug or siRNA resulted in robust induction of IL-36 $\gamma$ that was several fold higher than induction by EGFRi alone. The effect of $C$. acnes is mediated by TLR2, because combining EGFRi and TLR2 agonist treatment yielded a comparable effect, and TLR2 siRNA abrogated it. Further, this combinatorial treatment of normal human skin explants induced expression of IL-8 in an IL-36 $\gamma$-dependent manner, thus showing that simultaneous EGFR inhibition and TLR2 agonism synergistically stimulate keratinocytes to express IL-36 $\gamma$ and consequently IL-8, driving neutrophil recruitment to follicles and manifesting as an acneiform eruption (Figure 1) (3).

The follicular predilection of lesions in EGFRi/MEKi-induced eruptions is not unexpected given the major role EGFR plays in hair follicle morphogenesis (EGF dependency), first established in 1995 via a series of knockout mouse (6-8) and epidermal dominant-negative (9) studies. Mice showed curly, rudimentary whiskers and variably decreased and disoriented follicles with little to no hair outgrowth, eventuating in alopecia (9); these defects have since been shown to be largely cell autonomous rather than secondary to inflammation (10). Early conjecture regarding the etiology of the EGFRi-induced acneiform rash focused on dysmaturation of follicular keratinocytes and other components of the pilosebaceous unit, leading to follicular occlusion and consequently inflammation (11). However, this new connection to C. acnes Satoh et al. (3) identified provokes an alternative hypothesis, namely, that the lesions are folliculocentric because the lipophilic bacteria congregate in the sebum-laden environment of the follicle. 
A

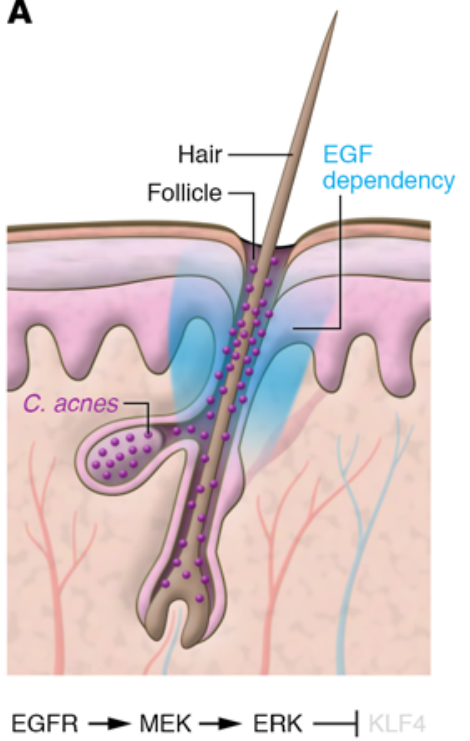

B

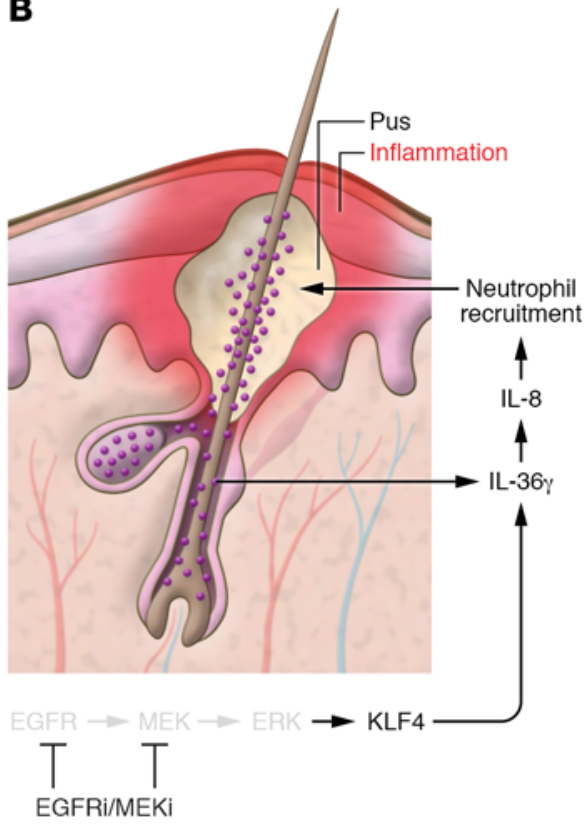

tions and pustular psoriasis, in which IL-36 factors critically (16), suggests that KLF4 may contribute to psoriasis pathogenesis through this previously unknown role in promoting IL-36 $\gamma$ expression.

\section{Murine models}

Mouse models have been indispensable for characterization of the EGFR/MEK/ERK pathway and as such continue to facilitate the exploration of EGFRi-induced adverse effects. However, Satoh et al. determined that mice lack the critical KLF4 binding site found in the human IL-36y promoter, and thus mouse keratinocytes fail to synergistically induce IL-36 $\gamma$ in response to EGFR inhibition and NF- $\mathrm{KB}$ activation (3). Additionally, others have found that, possibly because of in vivo compensation, adult EGFR-deficient mice showed ERK phosphorylation remains unchanged in keratinocytes $(17,18)$. These findings caution against the use of murine models for dissecting the mechanisms that underlie EGFRi-induced acneiform eruptions. Nonetheless, mice with EGFR ablation specific to epidermal keratinocytes have been described in several studies to exhibit enhanced and aberrant chemokine production and recruit mixed inflammatory infiltrate composed of macrophages, mast cells, neutrophils, and $\mathrm{T}$ cells (19, 20). These mice also showed abnormal epidermal differentiation with alteration in microbial defense gene expression (19,
Figure 1. A model for folliculocentric pustular drug eruption. (A) EGFR signaling is critical to hair follicle development and maintenance (EGF dependency). MEK and ERK1/2 signaling promotes degradation of transcription factor Krüppel-like factor 4 (KLF4). (B) During treatment with EGFR or MEK inhibitors, KLF4 accumulates and binds the IL36C promoter in cooperation with $\mathrm{NF}-\kappa \mathrm{B}$ activated by Cutibacterium acnes

(C. acnes) via TLR2 to drive IL-36 $\gamma$ expression. This in turn promotes IL-8 expression and consequent neutrophil recruitment and presents as a folliculocentric pustular drug eruption.
20), resulting in reduced bactericidal activity against Staphylococcus aureus that likely contributes to increased colonization of patients undergoing EGFRi therapy (20).

Further, the epidermal EGFR-knockout mice $(19,20)$ as well as mice treated with EGFRis (21) were found to show elevated IL-1 $\beta$ and TNF- $\alpha$ expression in skin, motivating the testing of an IL-1 receptor antagonist (anakinra) in mice undergoing antineoplastic treatment with EGFRis; however, anakinra appeared to reduce EGFRi antitumor activity (21). Because IL-36 is a member of the IL-1 superfamily and developing acneiform eruption during EGFRi therapy is a positive prognostic sign (22), assessing the impact of emerging therapies that inhibit IL-36 signaling (23) on EGFRi efficacy may be of interest. If EGFRi therapy represents a legitimate concern, it may be reasonable to target IL-36 $\gamma$ via topical therapies such as the vitamin $\mathrm{D}_{3}$ derivative calcipotriol, which was shown in a murine psoriasis model to inhibit IL-36a and IL-36 $\gamma$ expression in keratinocytes via the vitamin D receptor (24).

\section{Clinical implications}

Finally, in light of this new link to the commensal microorganism C. acnes, reactive and even preemptive treatment with the current mainstay - an oral tetracycline-class antibiotic such as doxycycline, alone or in combination with a topical antibiotic, corticosteroid, or retinoid - is 
now well justified. On the same note, the work by Satoh et al. (3) provides clarity regarding the superior benefit reported for full antimicrobial dosing of tetracyclines in treating EGFRi-induced pustular eruptions when compared with the lower doses often recommended nowadays for treatment of acne vulgaris and rosacea (25). Although subantimicrobial dosing in acne and rosacea patients still capitalizes on the pleiotropic antiinflammatory effects of tetracyclines with less selective pressure and thus lower risk of developing resistant organisms, the bacteriostatic effects of tetracyclines on $C$. acnes, rather than the antiinflammatory effects, may indeed truly benefit patients undergoing EGFRi therapy.

\section{Acknowledgments}

This work was supported by the National Institute of Arthritis and Musculoskeletal and Skin Diseases of the NIH (5T32AR007197-40 to ACB and R01AR069071 to JEG) as well as the A. Alfred Taubman Medical Research Institute's Taubman Institute Innovation Projects program, the Frances and Kenneth Eisenberg Emerging Scholar Award, and the University of Michigan Babcock Endowment Fund (to JEG).

Address correspondence to: Johann E. Gudjonsson, 1500 E. Medical Center Drive, Ann Arbor, Michigan, USA. Phone:734.353.0091; Email:johanng@med.umich.edu.

\footnotetext{
1. Agero AL, Dusza SW, Benvenuto-Andrade C, Busam KJ, Myskowski P, Halpern AC. Dermatologic side effects associated with the epidermal growth factor receptor inhibitors. J Am Acad
}

Dermatol. 2006;55(4):657-670.

2. Ranson M, et al. ZD1839, a selective oral epidermal growth factor receptor-tyrosine kinase inhibitor, is well tolerated and active in patients with solid, malignant tumors: results of a phase I trial. J Clin Oncol. 2002;20(9):2240-2250.

3. Satoh TK, et al. IL-36 $\gamma$ drives skin toxicity induced by EGFR/MEK inhibition and commensal Cutibacterium acnes. J Clin Invest. 2020;130(3):1417-1430.

4. Towne JE, Garka KE, Renshaw BR, Virca GD, Sims JE. Interleukin (IL)-1F6, IL-1F8, and IL-1F9 signal through IL-1Rrp2 and IL-1RAcP to activate the pathway leading to NF-kappaB and MAPKs. JBiol Chem. 2004;279(14):13677-13688.

5 . Kim J, et al. Activation of toll-like receptor 2 in acne triggers inflammatory cytokine responses. Jimmunol. 2002;169(3):1535-1541.

6. Miettinen PJ, et al. Epithelial immaturity and multiorgan failure in mice lacking epidermal growth factor receptor. Nature. 1995;376(6538):337-341.

7. Sibilia M, Wagner EF. Strain-dependent epithelial defects in mice lacking the EGF receptor. Science. 1995;269(5221):234-238.

8. Threadgill DW, et al. Targeted disruption of mouse EGF receptor: effect of genetic background on mutant phenotype. Science. 1995;269(5221):230-234.

9. Murillas R, Larcher F, Conti CJ, Santos M, Ullrich A, Jorcano JL. Expression of a dominant negative mutant of epidermal growth factor receptor in the epidermis of transgenic mice elicits striking alterations in hair follicle development and skin structure. EMBO J. 1995;14(21):5216-5223.

10. Amberg N, et al. EGFR controls hair shaft differentiation in a p53-independent manner. iscience. 2019;15:243-256.

11. Journagan S, Obadiah J. An acneiform eruption due to erlotinib: prognostic implications and management. JAm Acad Dermatol. 2006;54(2):358-360.

12. Campbell $\mathrm{P}$, et al. Epithelial inflammation resulting from an inherited loss-of-function mutation in EGFR. J Invest Dermatol. 2014;134(10):2570-2578.

13. Segre JA, Bauer C, Fuchs E. Klf4 is a transcription factor required for establishing the barrier function of the skin. Nat Genet. 1999;22(4):356-360.
14. Sen GL, et al. ZNF750 is a p63 target gene that induces KLF4 to drive terminal epidermal differentiation. Dev Cell. 2012;22(3):669-677.

15. Li B, et al. Transcriptome analysis of psoriasis in a large case-control sample: RNA-seq provides insights into disease mechanisms. J Invest Dermatol. 2014;134(7):1828-1838.

16. Johnston A, et al. IL-1 and IL-36 are dominant cytokines in generalized pustular psoriasis. J Allergy Clin Immunol. 2017;140(1):109-120.

17. Nagao K, Kobayashi T, Ohyama M, Akiyama H, Horiuchi K, Amagai M. Brief report: requirement of TACE/ADAM17 for hair follicle bulge niche establishment. Stem Cells. 2012;30(8):1781-1785

18. Wolf C, Qian Y, Brooke MA, Kelsell DP, Franzke CW. ADAM17/EGFR axis promotes transglutaminase-dependent skin barrier formation through phospholipase $\mathrm{C} \gamma 1$ and protein kinase $\mathrm{C}$ pathways. Sci Rep. 2016;6:39780.

19. Mascia F, et al. Genetic ablation of epidermal EGFR reveals the dynamic origin of adverse effects of anti-EGFR therapy. Sci Transl Med. 2013;5(199):199ra110.

20. Lichtenberger BM, et al. Epidermal EGFR controls cutaneous host defense and prevents inflammation. Sci Transl Med. 2013;5(199):199ra111.

21. Surguladze D, et al. Tumor necrosis factor-alpha and interleukin-1 antagonists alleviate inflammatory skin changes associated with epidermal growth factor receptor antibody therapy in mice. Cancer Res. 2009;69(14):5643-5647.

22. Pérez-Soler R. Can rash associated with HER1/ EGFR inhibition be used as a marker of treatment outcome? Oncology (Williston Park, NY). 2003;17(11 suppl 12):23-28

23. Bachelez H, et al. Inhibition of the interleukin-36 pathway for the treatment of generalized pustular psoriasis. N Engl J Med. 2019;380(10):981-983.

24. Germán B, et al. Disrupting the IL-36 and IL-23/ IL-17 loop underlies the efficacy of calcipotriol and corticosteroid therapy for psoriasis. JCI Insight. 2019;4(2):123390.

25. Vaubel J, Livingstone E, Schadendorf D, Zimmer L. Retarded low-dose doxycycline for EGFR or MEK inhibitor-induced papulopustular rash. J Eur Acad Dermatol Venereol. 2014;28(12):1685-1689. 